\title{
P-0433: Investigating Hypoglycemic Effects of the Herbal Drug Jiang Tang 2016 (J T2016).
}

Brown Mary Nthabeleng ${ }^{1}$, Ma Xuesheng ${ }^{2}$, Wei Ying ${ }^{3,5}$, Qing Tianyu ${ }^{3,4}$, Dietrich Danee ${ }^{1}$, Xu Tunhai ${ }^{3,5}$, Liu Tonghua $a^{3,4}$

1. Department of Medical Bioscience, University of the Western Cape, Cape Town 7535, South Africa;

2. School of Natural Medicine, University of the Western Cape, Cape Town 7535, S outh Africa;

3. Health-Cultivation Laboratory of the Ministry of Education, Beijing University of Chinese Medicine, Beijing 100029, China;

4. School of Graduates, Beijing University of Chinese Medicine, Beijing 100029, China;

5. School of Chinese Herb Pharmacy, Beijing University of Chinese Medicine, Beijing 100029, China;

Correspond author: LIU Tonghua, Beijing University of Chine se Medicine, Beijing 100029, China. Email: thliu@ vip.163.com.

This research is sponsored by International Cooperation Ministry of Science and Technology China (No.2012DFG31550)

\section{Aim}

The aim of this study was to investigate the anti-diabetic effects of the herbal drug compound, JT2016 in HFD- STZ induced diabetic SD rats.

\section{Objectives}

$>$ To determine the hypoglycemic effects of JT2016 in vivo.

$>$ To investigate the biological mechanism of the anti-diabetic effects of JT2016, and discover its most effective compound.

\section{Materials and Methods}

Sixty (60) male, seven weeks normal Sprague Dawley (SD) rats (210$260 \mathrm{~g}$ ) were used for the study. All animals were housed at $23+/-25^{\circ} \mathrm{C}$ temperature, humidity of $55+/-10 \%$ with a 12 -hour light/dark cycle, and were provided with food and water ad libitum. Following two weeks of adaptive feeding, the rats were divided into two main groups; the normal group - fed with normal pellet diet (NPD) ( $n=10)$, and the experimental group - fed with High Fat Diet (HFD) ( $n=50)$.

HFD rats were injected intraperitoneally with low dose Streptozotocin (STZ) $(35 \mathrm{mg} / \mathrm{kg})$. Three days later, the animals were fasted and tested for hyperglycemia; every rat tested positive for hyperglycemia $(+16$ $\mathrm{mmol} / \mathrm{L}$ ) was included in the study and randomly assigned into the following five groups: control group, positive drug group (pioglitazone $500 \mathrm{mg} / \mathrm{kg}$ ), JT2016 compound groups: JT2016A group (300mg/kg), JT2016B (300mg/kg), and JT2016C group $(300 \mathrm{mg} / \mathrm{kg})$. All medicine was administered intragastrically. Body weight, fasting blood glucose(FBG) and random blood glucose (RBG) were measured weekly for six weeks of treatment. At the end of the six weeks, the animals were tested for FBG, plasma insulin (PI), tumour necrosis factor alpha (TNF- $\alpha$ ), interleukin factor six (IL-6) and executed through anesthesia.

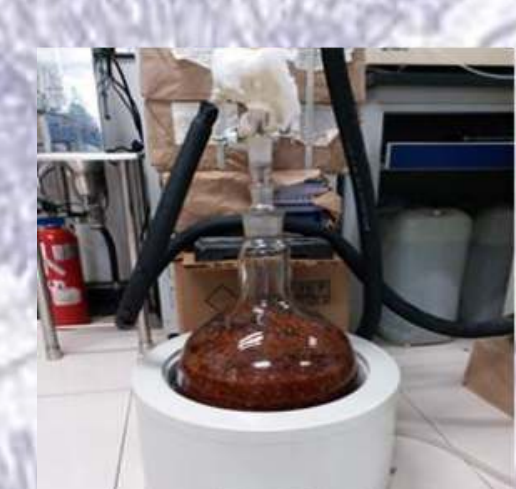

몽-9

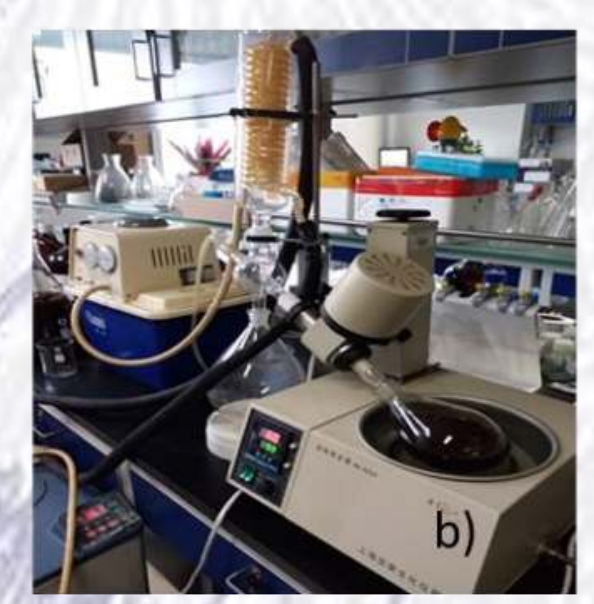

b)

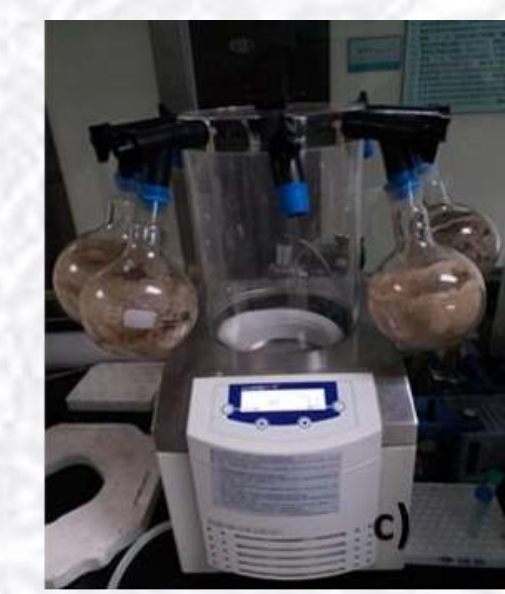

Figure 1. Preparation of the herbal compound JT2016 at the HealthCultivation Laboratory, Beijing University of Chinese Medicine (a: extraction, b: concentration, c: freeze drying.)

\section{Results}

JT2016C significantly $(p<0.05)$ lowered and normalized the blood glucose levels in HFD-fed diabetic SD rats as compared to the control group. It also significantly $(p<0.05)$ lowered FBG, PI, IL-6, and TNF-a levels as compared to the control group, and proved to be better and more effective as to the positive drug, JT2016A, and JT2016B groups.
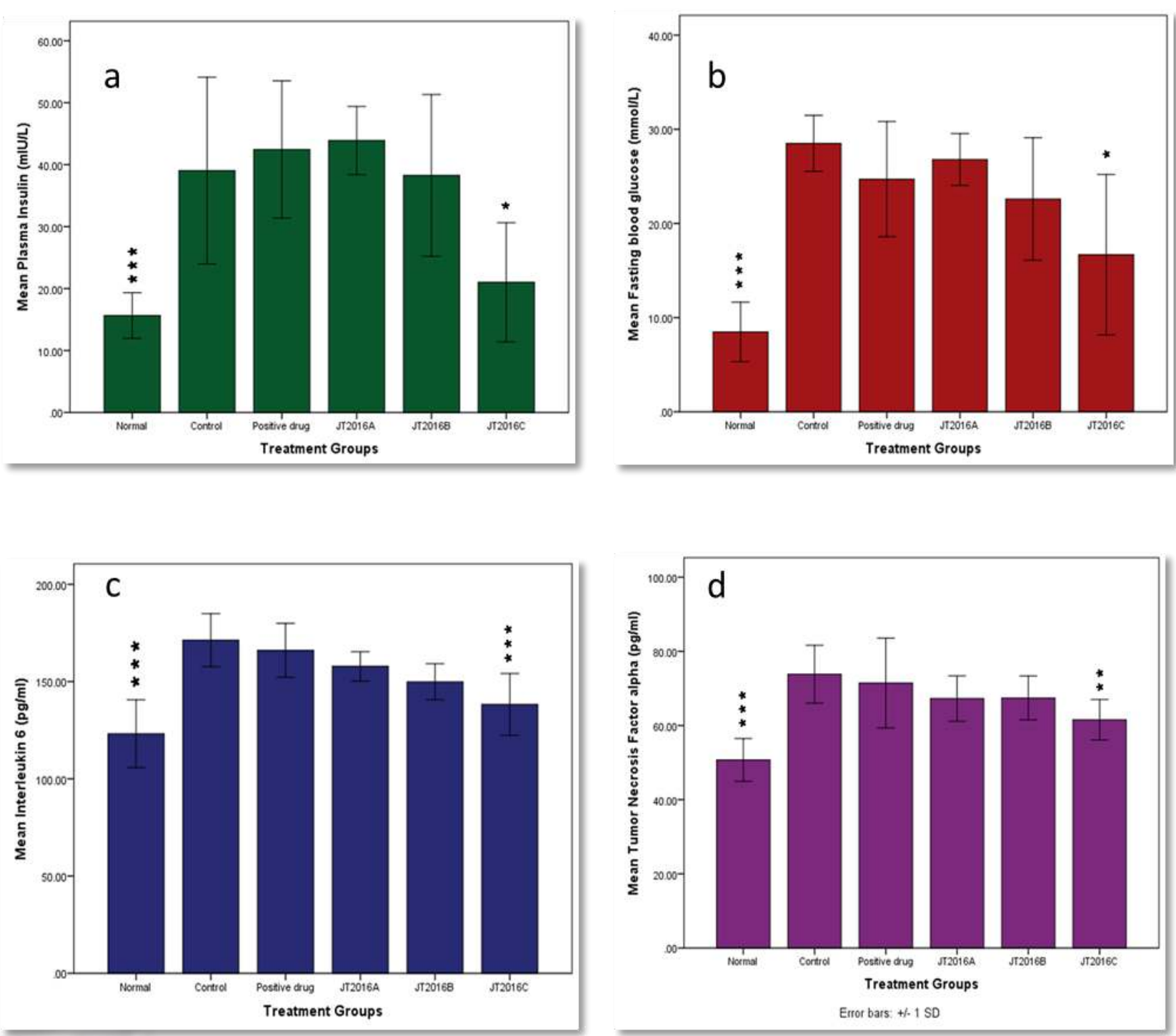

Figure 2. The effect of treatment groups on (a) Plasma insulin (b) fasting blood glucose, (c) IL-6, (d) TNF- $\alpha$ in HFD - STZ induced diabetic SD rats. Values are mean $+/-$ SD. ${ }^{*} p<0.05$ vs control group $(n=9), * * p$ $<0.001$ vs control group, and ${ }^{* * *} p<0.0001$ vs control group.

\section{Conclusion}

JT2016C demonstrates positive anti-diabetic effects in HFD-STZ induced diabetic rats as compared to other treatment groups. Therefore, this study can be used as a stepping stone to develop JT2016 into an effective anti-diabetes herbal drug for T2DM management, with further in vivo and invitro investigations.

\section{Acknowledgements}

International Cooperation Ministry of Science and Technology, China International Relations Office, University of the Western Cape, South Africa. 